(2)

\title{
Visual field changes as an early indicator of glioblastoma multiforme progression: two cases of functional vision changes before MRI detection
}

This article was published in the following Dove Press journal:

Clinical Ophthalmology

II June 2015

Number of times this article has been viewed

Kate $X i e^{1, *}$

Catherine $\mathrm{Y} \mathrm{Liu}^{1, *}$

Anton $\mathrm{N} \mathrm{Hasso}^{2}$

Robert Wade Crow'

'Department of Ophthalmology, Gavin Herbert Eye Institute, University of California, Irvine, Irvine, CA, USA, ${ }^{2}$ Department of Radiological Sciences, University of California Irvine Medical Center, Orange, CA, USA

*These authors contributed equally to this work
Correspondence: Robert Wade Crow Gavin Herbert Eye Institute, Department of Ophthalmology, University of California, Irvine, 850 Health Sciences Road, Irvine, CA 92697, USA

$\mathrm{Tel}+\mathrm{l} 9498240158$

Fax + I 9498244015

Email rcrow@uci.edu

\begin{abstract}
Glioblastoma multiforme is an aggressive tumor associated with a high rate of recurrence even after maximal therapy. In a disease with poor prognosis and rapid deterioration, early detection of tumor progression is necessary to make timely treatment decisions or to initiate end of life care. We identify two cases where Humphrey visual field testing predated magnetic resonance imaging and positron emission tomography findings of tumor progression by months in glioblastoma multiforme. New or worsening visual field defects may indicate signs of tumor progression in glioblastoma multiforme and should prompt further investigation.
\end{abstract}

Keywords: glioblastoma multiforme, optic pathway, visual field defects

\section{Introduction}

Detection of tumor progression in glioblastoma multiforme (GBM) has been largely dependent on improved imaging interpretation. However, this remains a challenge. High grade gliomas display irregular growth and enhancement patterns. Following treatment, it is difficult to differentiate pseudoprogression, an increase in contrast enhancement due to treatment effect, from true progression using conventional imaging techniques. ${ }^{1-3}$ Currently, the MacDonald and updated Response Assessment in Neuro-Oncology (RANO) criteria for malignant gliomas consider a combination of imaging and clinical findings to assess treatment response. ${ }^{4}$ The description of clinical deterioration in these criteria has been purposely left vague, due to the highly variable neurologic presentations of these tumors and the difficulty of objectively measuring a patient's subjective experience. ${ }^{4,5}$ Neurocognitive function testing and quality of life assessment have been proposed as possible methods to improve measurement of clinical status. ${ }^{4,5}$

We present two case studies where Humphrey visual field (HVF) testing proved to be a useful objective tool to assess deterioration of visual function in patients with GBM occurring near the optic pathways. In both cases, HVF testing revealed visual field defects correlating to tumor spread months before structural changes were seen on magnetic resonance imaging (MRI).

\section{Case presentation Case I}

A 53-year-old male presented with right-sided blurry vision and intermittent monocular diplopia. He had a history of GBM (World Health Organization [WHO] grade IV) of the left parietal lobe and had undergone surgical resection, radiation, and concomitant temozolomide chemotherapy 7 months prior to presentation. At the time of evaluation in the neuro-ophthalmology clinic, he was being treated with adjuvant temozolomide 
chemotherapy and was enrolled in the Celldex ACT IV trial (NCT01480479) after testing positive for the EGFRv3 mutation. His past ocular history was significant for laser-assisted in situ keratomileusis (LASIK) procedures in both eyes and dry eye syndrome. His vision had been stable until a few months prior, when he described intermittent blurry vision and monocular diplopia of the right eye.

On examination, visual acuity was $20 / 40+2$ with pinhole correction to $20 / 30$ on the right and $20 / 25+1$ with no pinhole improvement on the left. Intraocular pressures were within normal range, and there was no afferent pupillary defect. Extraocular motility was full, and the patient denied binocular diplopia. Slit lamp examination revealed blepharitis in both eyes. Dilated fundus exam showed no irregularities of the optic disc, macula, or vessels. Optical coherence tomography of the nerve fiber layer revealed mild retinal nerve fiber layer thinning in both eyes. HVF testing showed nonspecific incongruous inferior field changes in the right eye (Figure 1).
A

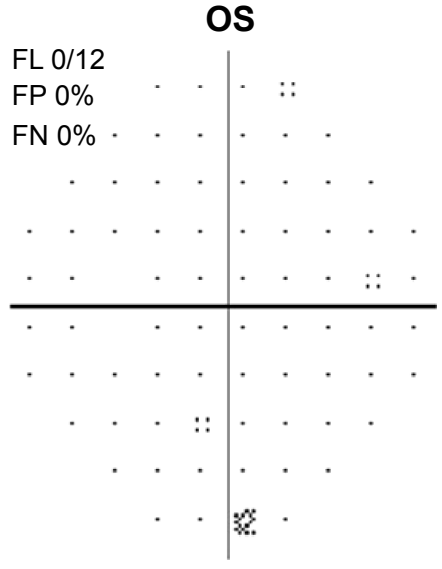

B

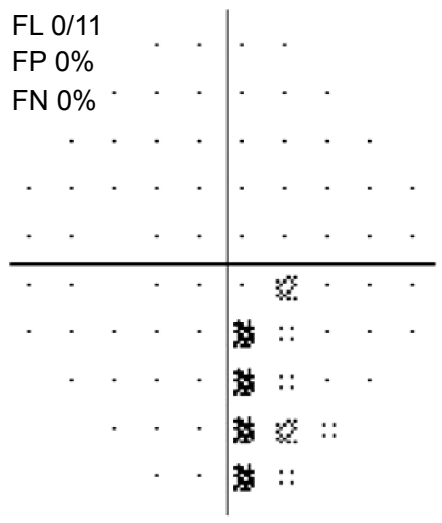

C

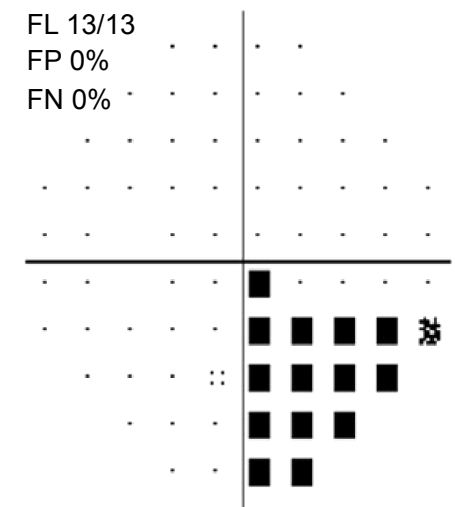

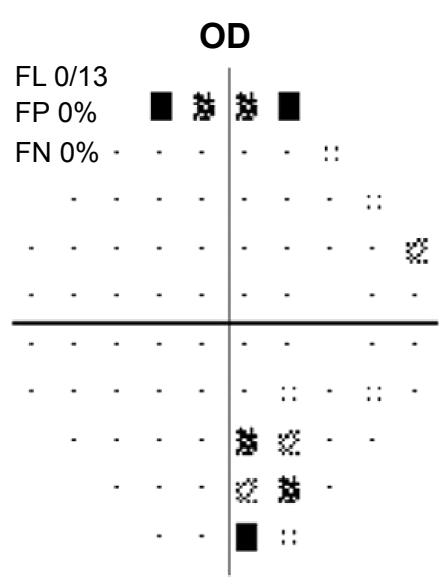
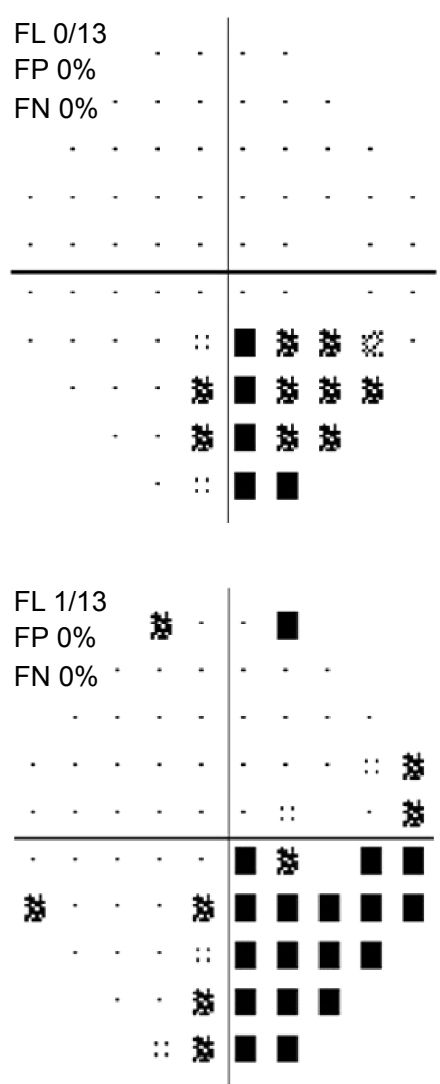

Figure I Humphrey visual field stimulus III 24-2 pattern deviation plots for case I.

Notes: (A) At time of presentation, (B) 10 weeks and 6 days later, and (C) 14 weeks and 2 days from time of presentation. Abbreviations: FL, fixation loss; FP, false positive; FN, false negative. 
The patient was managed conservatively, with treatment of anterior surface disease and a plan to monitor visual fields. MRI showed a decreasing tumor size, likely related to chemotherapy effect (Figure 2).

Eleven weeks later, HVF revealed incongruous right homonymous inferior quadrantanopia (Figure 1). Positron emission tomography (PET) and MRI scans were obtained to assess for progression of disease, and neither showed indication of progressive disease (Figure 2). At his 14 week follow-up, visual acuity was $20 / 60$ on the right and 20/25 on the left. No relative afferent pupillary defect was present. Slit lamp examination was unchanged. HVF testing showed worsening right homonymous inferior quadrantanopia (Figure 1). Repeat MRI 1 week later, a total of 4 weeks from definitive identification of his right homonymous inferior field defect, showed significant interval increase in size, enhancement, and vasogenic edema in the region of the tumor (Figure 2).

The patient passed away 2 months later, 17 months from the time of his initial diagnosis.
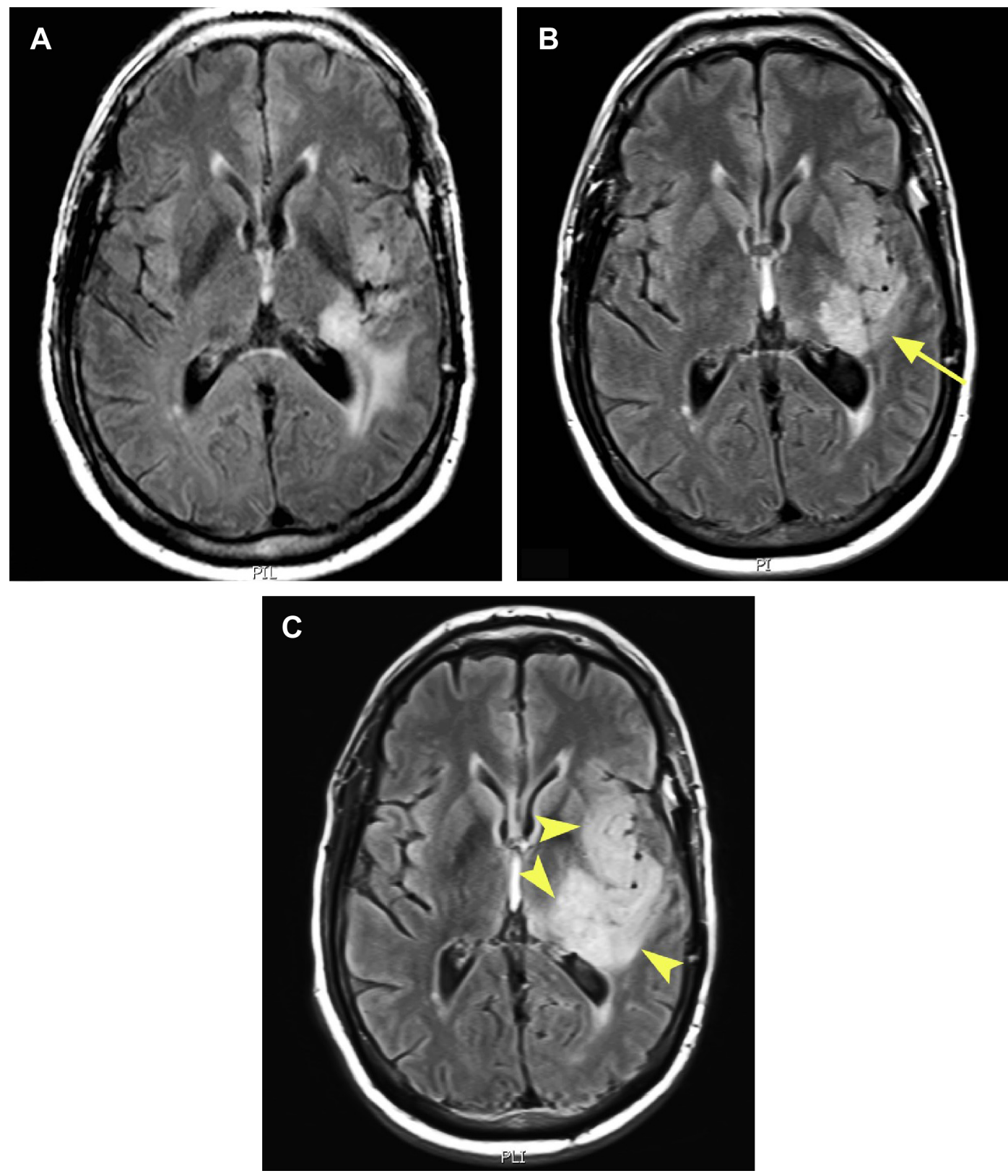

Figure 2 Axial FLAIR T2 (TR:500, TI:2500, TE:I25) weighted MRI images for case I taken at approximately the same levels in the area of the mid-thalamus.

Notes: (A) I week after time of presentation, showing tumor involvement at the subinsular, thalamic, and along the posterior aspect of the left lateral ventricle. (B) 9 weeks and 6 days after time of presentation, with interval decrease in tumor enhancement along the left lateral ventricle (arrow). (C) I4 weeks and 3 days after time of presentation, showing interval progression with widespread involvement of the left periventricular region, thalamus, and insular cortex (arrowheads). 


\section{Case 2}

A 53-year-old man presented with intermittent blurry vision and diplopia. He had GBM (WHO stage IV) of the left parietal lobe and had undergone two surgical resections, two courses of radiation therapy, and multiple chemotherapy regimens including temozolomide, irinotecan (Camptothecin-11, CPT-11), and bevacizumab, and at time of evaluation he was being treated with carboplatin/bevacizumab along with NovoTTF-100A therapy. The patient had been seen in the neuro-ophthalmology clinic for 6 months prior to this visit for blurry vision, which was correctable by refraction. His previous HVF showed incongruous nonspecific defects (Figure 3). On initial exam, best-corrected visual acuity was 20/25 in both eyes. Intraocular pressures were within normal range. Extraocular motility was full in all gazes. No relative afferent pupillary defect was present. Fundus examination of both eyes was unremarkable.

Repeat HVF on this visit showed a new right homonymous hemianopsia (Figure 3). Given these findings, an MRI
A

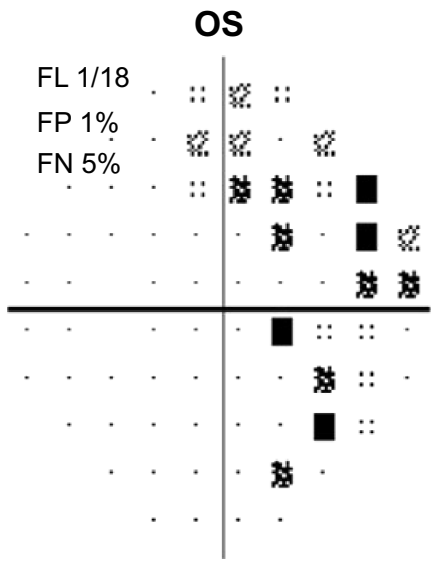

B
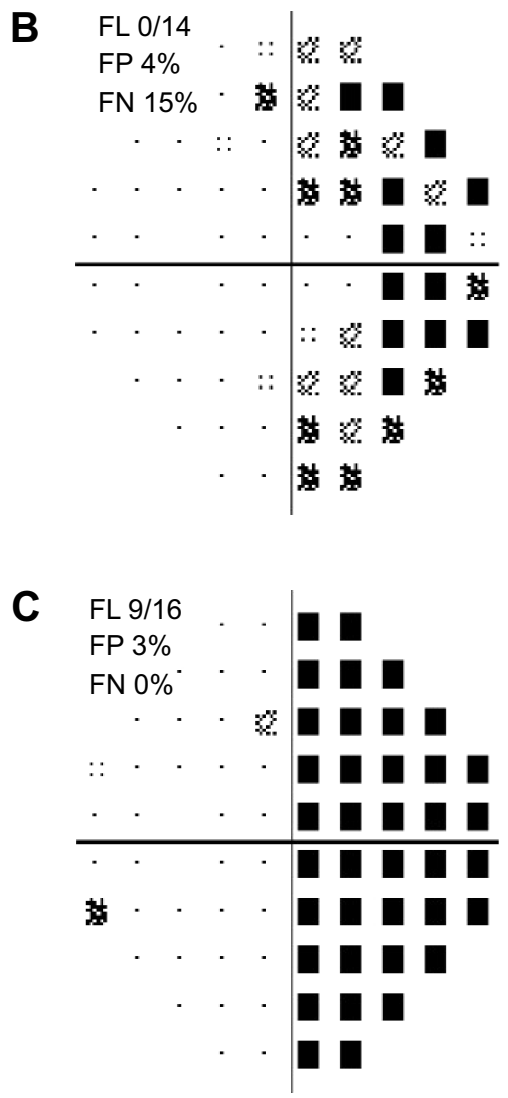
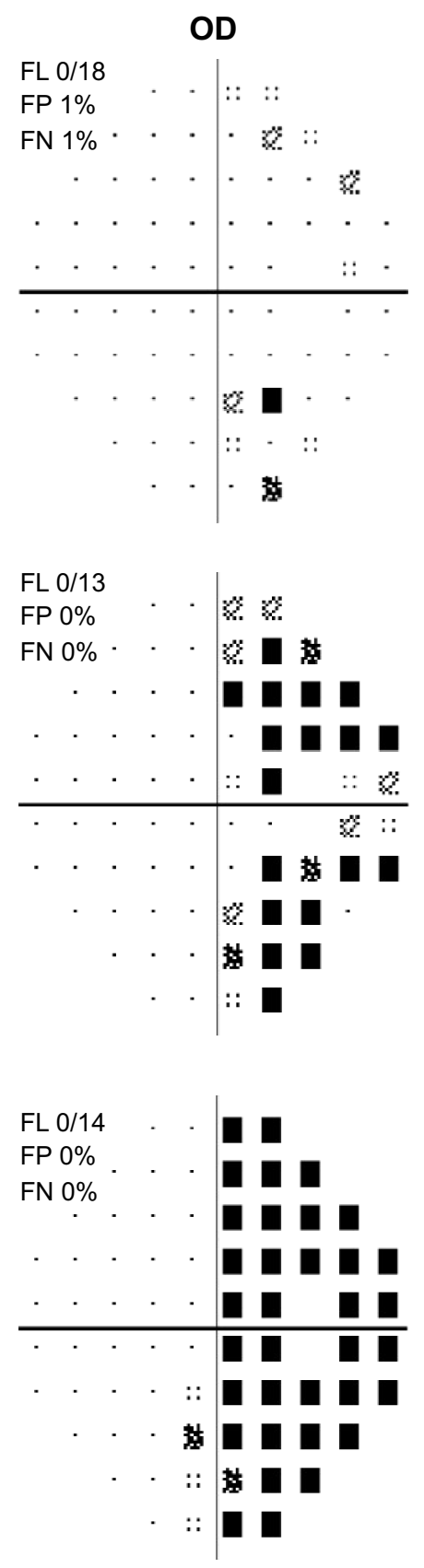

Figure 3 Humphrey visual field stimulus III 24-2 pattern deviation plots for case 2 .

Notes: (A) Baseline, II weeks and 5 days prior to onset of visual changes, (B) time of presentation, and (C) I 3 weeks and I day after time of presentation. Abbreviations: FL, fixation loss; FP, false positive; FN, false negative. 
brain scan was ordered to evaluate for possible tumor progression. Interestingly, it showed a decrease in tumor intensity, which was attributed to treatment response (Figure 4). No evidence of tumor progression was observed.

Thirteen weeks from that initial change seen on HVF, the patient continued to complain of worsening vision. Visual acuity was stable at 20/25 in both eyes. A repeat HVF showed worsening right homonymous hemianopsia (Figure 3). MRI from 2 days earlier showed stable size of known tumors (Figure 4). Four weeks later, a total of 4 months after definitive worsening of HVF had been identified, MRI showed definitive tumor progression with increased size/ enhancement of disease involving the midbrain, right thalamus, left medial temporal lobe, and left anterior medulla and dorsal column of the proximal spinal cord (Figure 4). In the neuro-oncology clinic, the patient described worsening vision and balance difficulties. Another cycle of lomustine (CCNU) and bevacizumab was started the following day.

Subsequent imaging continued to show tumor progression and the patient continued to deteriorate clinically.
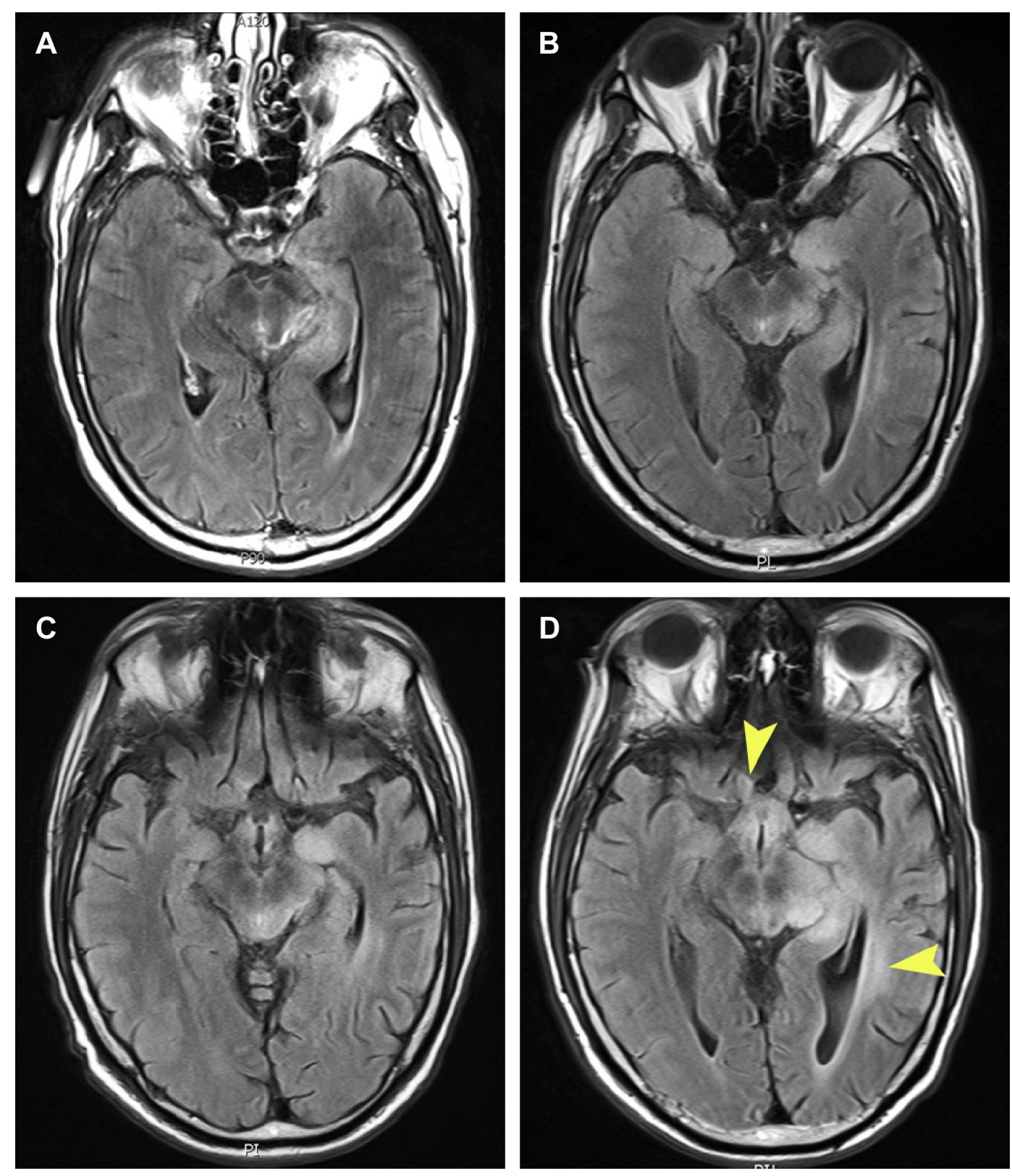

Figure 4 Axial FLAIR T2 (TR:500, TI:2500, TE:125) weighted MRI images taken at approximately the same levels in the region of the midbrain for case 2.

Notes: (A) Baseline, II weeks and 4 days prior to onset of visual changes, showing tumor involvement of the left hippocampus, midbrain, and posterior cingulate. (B) Time of presentation, with no significant change from baseline. (C) 12 weeks after time of presentation, with no significant interval change from previous. (D) I6 weeks after time of presentation, showing progressive disease anteriorly to the optic nerves and chiasm and posteriorly along the optic radiations (arrowheads). 
The patient ultimately decided to withdraw care and passed away 2 months later, 24 months from initial diagnosis.

\section{Discussion}

We describe definitive visual field defects preceding MRI findings in progressive GBM by 1 month in case 1 and 4 months in case 2. Clinical evidence of progression can precede MRI evidence in both initial and recurrent GBM, with seizures being the most common preceding symptom. ${ }^{6,7}$ However, to our knowledge, this is the first report of clear, progressive visual field defects predating neuroimaging identification of tumor progression.

Following chemoradiotherapy, the irregular enhancement and growth patterns of GBM make it difficult to differentiate early tumor progression from pseudoprogression or radiation necrosis on neuroimaging. ${ }^{2,3,8}$ The updated RANO criteria address this dilemma with more stringent imaging criteria on conventional MRI for tumor response. ${ }^{4}$ Ultimately, the resolution of MRI imaging remains limited, as seen in our two cases, where MRI was heavily relied upon to define tumor borders. For example, in a study of preoperative MRI prediction of histopathologic margins of optic nerve glioma resection, $27 \%$ of patients showed evidence of microscopic tumor involvement at margins posterior to those indicated on preoperative MRI. ${ }^{9}$ Recognizing the limitations of conventional MRI, more advanced methods such as magnetic resonance spectroscopy, diffusion MRI, perfusion MRI, and amino acid PET are currently being investigated as adjunctive measures to differentiate treatment response from tumor progression in GBM. ${ }^{10,11}$ In a recent systematic review of imaging techniques to detect glioblastoma progression, the authors comment on the wide-ranging results in the literature, in part due to the lack of a consensus on the neuroimaging definition of tumor progression. ${ }^{1}$

HVF testing is a functional study that adds sensitivity to detecting disease progression of tumors known to involve the optic pathway. Functional studies provide a clinical context for imaging findings, increasing the predictive value of a positive imaging finding. This fundamental concept is well known but often overlooked in the era of increasingly sophisticated imaging techniques. Overreliance on imaging that does not fit with clinical findings may lead to delayed treatment, inappropriate treatment, or unnecessary tests. ${ }^{12}$ While imaging has played and will continue to play a key role in the monitoring of GBM, the development of accurate tools to assess clinical status should be similarly emphasized. HVF testing may prove to be useful for monitoring clinical signs of progression, as up to $50 \%$ of patients with lesions in the optic pathway show visual field defects. ${ }^{13}$ The utility of HVF testing has already been recognized in the area of hydroxychloroquine toxicity screening, where roughly $10 \%$ of patients manifest visual field changes in a classic ring scotoma pattern before any evidence of photoreceptor damage on optical coherence tomography macula and fundus exam. ${ }^{14}$ While HVF testing can be prone to error, well documented, reliable studies that show a clear pattern of visual changes can alert clinicians to prompt workup.

There are some limitations to our review and case report. For example, in case 2, the patient received radiation treatment 1 month prior to presentation. A visual field deficit resulting from irradiation to the optic pathways could have been a plausible initial explanation for the patient's presentation. However, his worsening visual field deficits on follow-up examination were ultimately predictive of MRI findings consistent with tumor progression. As we describe only two cases, additional studies are needed to determine the specificity and sensitivity of HVF testing, to identify appropriate referral criteria for visual field testing, and to specify follow-up criteria once a visual field deficit is noted. Ultimately, we must also recognize that the diagnosis of GBM progression can only be definitively made by biopsy with histologic identification.

In the absence of highly accurate and early neuroimaging identification of tumor progression, HVF testing is useful as an adjunctive clinical evaluation. In our two cases, HVF showed clear, progressive homonymous field defects in the setting of visual complaints and negative MRI findings. Therefore, for rapidly growing tumors occurring near optic pathways, such as glioblastoma, we recommend prompt neuro-ophthalmological evaluation with HVF testing. Evidence of clear progressive visual field deficits requires mandatory clinical monitoring and should prompt further systemic evaluation and consideration of changes in treatment regimens. HVF testing may very well be a first step toward bridging the gap between functional and imaging identification of tumor progression in GBM involving the optic pathways.

\section{Disclosure}

The authors report no conflicts of interest in this work.

\section{References}

1. Ryken TC, Aygun N, Morris J, et al. The role of imaging in the management of progressive glioblastoma: a systematic review and evidencebased clinical practice guideline. J Neurooncol. 2014;118(3):435-460.

2. O'Brien BJ, Colen RR. Post-treatment imaging changes in primary brain tumors. Curr Oncol Rep. 2014;16(8):397. 
3. Mehta AI, Kanaly CW, Friedman AH, Bigner DD, Sampson JH. Monitoring radiographic brain tumor progression. Toxins (Basel). 2011; 3(3):191-200.

4. Wen PY, Macdonald DR, Reardon DA, et al. Updated response assessment criteria for high-grade gliomas: response assessment in neurooncology working group. J Clin Oncol. 2010;28(11):1963-1972.

5. Reardon DA, Galanis E, DeGroot JF, et al. Clinical trial end points for high-grade glioma: the evolving landscape. Neuro Oncol. 2011;13(3): 353-361.

6. Landy HJ, Lee TT, Potter P, Feun L, Markoe A. Early MRI findings in high grade glioma. J Neurooncol. 2000;47(1):65-72.

7. Chittiboina P, Connor DE Jr, Caldito G, Quillin JW, Wilson JD, Nanda A. Occult tumors presenting with negative imaging: analysis of the literature. J Neurosurg. 2012;116(6):1195-1203.

8. Jahangiri A, Aghi MK. Pseudoprogression and treatment effect. Neurosurg Clin N Am. 2012;23(2):277-287, viii-ix.

9. Spicer GJ, Kazim M, Glass LR, et al. Accuracy of MRI in defining tumorfree margin in optic nerve glioma surgery. Ophthal Plast Reconstr Surg. 2013;29(4):277-280.
10. Reardon DA, Ballman KV, Buckner JC, Chang SM, Ellingson BM. Impact of imaging measurements on response assessment in glioblastoma clinical trials. Neuro Oncol. 2014;16 Suppl 7:vii24-vii35.

11. Kim JH, Choi SH, Ryoo I, et al. Prognosis prediction of measurable enhancing lesion after completion of standard concomitant chemoradiotherapy and adjuvant temozolomide in glioblastoma patients: application of dynamic susceptibility contrast perfusion and diffusion-weighted imaging. PLoS One. 2014;9(11):e113587.

12. Sadun AA, Chu ER, Boisvert CJ. Neuro-ophthalmology Safer Than MRI? Ophthalmology. 2013;120(4):879.

13. Kedar S, Ghate D, Corbett JJ. Visual fields in neuro-ophthalmology. Indian J Ophthalmol. 2011;59(2):103-109.

14. Marmor MF, Melles RB. Disparity between visual fields and optical coherence tomography in hydroxychloroquine retinopathy. Ophthalmology. 2014;121(6):1257-1262.
Clinical Ophthalmology

\section{Publish your work in this journal}

Clinical Ophthalmology is an international, peer-reviewed journal covering all subspecialties within ophthalmology. Key topics include: Optometry; Visual science; Pharmacology and drug therapy in eye diseases; Basic Sciences; Primary and Secondary eye care; Patien Safety and Quality of Care Improvements. This journal is indexed on

Submit your manuscript here: http://www.dovepress.com/clinical-ophthalmology-journal

\section{Dovepress}

PubMed Central and CAS, and is the official journal of The Society of Clinical Ophthalmology (SCO). The manuscript management system is completely online and includes a very quick and fair peer-review system, which is all easy to use. Visit http://www.dovepress.com/ testimonials.php to read real quotes from published authors. 